\title{
ACE inhibitors preserve cognitive function
}

In a mouse model of brain pathology mediated by a subset of anti-DNA antibodies found in patients with systemic lupus erythematosus (SLE), ACE inhibition can preserve cognitive function, according to a new study. "The most important aspect of the study is that the results showed that damaged neurons can recover and cognition can be improved if microglial activation is controlled," reports Betty Diamond, corresponding author of the study.

Her group has previously demonstrated that this subset of anti-DNA antibodies, which they term DNRAbs, cross-react with the $N$-methyl-D-aspartate receptor (NMDAR). "We have been studying how and when these antibodies can contribute to the neuropsychiatric symptoms of SLE," says Diamond. They developed a two-stage mouse model of neuropsychiatric lupus, in which DNRAbs are first induced by immunizing with a DWEYS peptide and the blood-brain barrier (BBB) is then disrupted using LPS treatment, resulting in acute excitotoxic neuron loss followed by long-term alterations in neuronal integrity and by spatial memory impairment.

In this study, Diamond and colleagues sought to understand the mechanisms underlying the longterm cognitive impairment in this model. "We demonstrate that the antibody-mediated neuronal death leads to microglial cell activation, and that microglial suppression can improve neuronal integrity and cognitive problems," explains Diamond.

Using C1q-deficient mice, they showed that the decrease in dendritic complexity and spine density, but not the acute neuronal death, was dependent on C1q. Unlike wildtype $\mathrm{DNRAb}^{+}$mice, $\mathrm{Clq}$-deficient $\mathrm{DNRAb}^{+}$mice behaved normally in an object-place memory task that tests spatial memory.

ACE inhibitors, commonly used to lower blood pressure, have

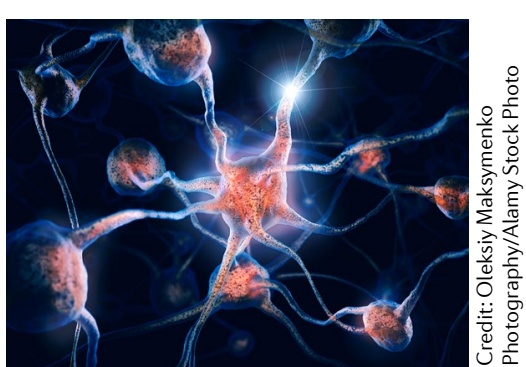

previously been shown to suppress microglial activation. In the new study, treatment with a BBB-

Treatment with a BBBpermeable ACE inhibitor... suppressed microglial activation and preserved dendritic integrity and cognitive function in the DNRAb ${ }^{+}$mice permeable ACE inhibitor, but not with a BBB-impermeable ACE inhibitor or saline, suppressed microglial activation and preserved dendritic integrity and cognitive function in the $\mathrm{DNRAb}^{+}$mice.

"We plan to study the mechanisms for microglial activation, which might lead to therapeutic targets" says Diamond. "We also think clinical trials of ACE inhibitors in neuropsychiatric SLE are now warranted."

Jessica McHugh

ORIGINAL ARTICLE Nestor, J. et al. Lupus

antibodies induce behavioral changes mediated by microglia and blocked by ACE inhibitors.J. Exp. Med. https://doi.org/10.1084/jem.20180776 (2018)

\section{RANKL reverse signalling and bone}

New research by Hiroshi Suzuki and colleagues shows that reverse signalling of receptor activator of nuclear factor- $\kappa \mathrm{B}$ (RANK; also known as TNFRSF11A) ligand (RANKL; also known as TNFSF11) in osteoblasts has a role in linking bone resorption and formation.

RANKL belongs to the tumour necrosis factor family, which is a group of proteins that act as bidirectional signalling molecules and produce intracellular reverse signalling. Previously, osteoblasts were thought to be the main producers of RANKL; however, mounting evidence suggests that osteocytes are the main source during bone remodelling.

"Because of this finding, we hypothesized that osteoblastic RANKL might have a different physiological role than that of osteocytic RANKL," explains corresponding author

Masashi Honma.

In this study, the researchers showed that maturing osteoclasts secreted vesicular RANK (vRANK), which binds to osteoblastic RANKL and activates reverse signalling through $R u n \times 2$. The stimulation also increased the mineralization of osteoblasts.

Next, the authors created a mouse model (Rankl ${ }^{P 29 A}$ ) to suppress reverse signalling of RANKL but not forward signalling, which triggers osteoclastogenesis. When recombinant RANKL was administered, osteoclast maturation in both Rankl ${ }^{P 29 A}$ and wild-type mice was increased; however, bone formation was disrupted in Rankl ${ }^{P 29 A}$ mice compared with wild-type mice. The results suggest that osteoblastic RANKL reverse signalling is involved in linking bone resorption and formation.

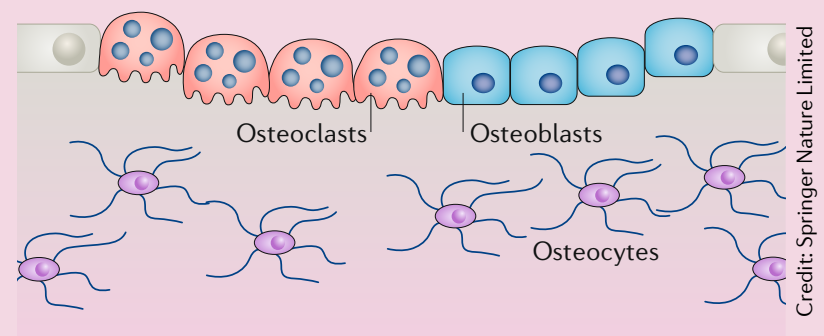

Finally, Suzuki and colleagues targeted RANKL reverse signalling in an ovariectomized mouse model to test whether it is a possible pharmacological target for the treatment of osteoporosis. They found that activation of RANKL reverse signalling inhibited reduced bone formation. "Our findings indicate that the role of RANKL is the accelerator of bone turnover rather ...activation of than the stimulator of bone resorption," concludes Honma.

Ivone Leong,

signalling inhibited reduced bone formation Nature Reviews Endocrinology This article is modified from the original in Nat. Rev. Endo. (https://doi.org/10.1038/s41574-018-0094-1).

ORIGINAL ARTICLE lkebuchi, Y. et al. Coupling of bone resorption and formation by RANKL reverse signalling. Nature 561, 195-200 (2018) 